\title{
Biochemistry and genetics: checks and balances
}

To study such complicated phenomena as transcriptional activation, biologists turn to two tried and true approaches - the biochemical and the genetic.

Biochemists use a strategy of reconstruction. They seek to reconstitute biological processes in test tubes. The first step is to develop an assay that reveals the activity of interest. The second is to purify the components of the cell necessary to reconstitute that activity from crude cell extracts. Finally, the purified proteins are studied individually and together to determine what each one does. Most of the three-dozen proteins required for transcription of class II genes were identified biochemically ${ }^{2,10,11}$.

Geneticists, on the other hand, employ a strategy of destruction. They disrupt biological processes by making mutations and examining the consequences in living cells. The first step is to develop a screen for cells exhibiting disruption in the process being studied (transcription, pattern formation, signal transduction or whatever). The second is to pinpoint any genetic mutations associated with that disruption, then further characterize the genes concerned to determine the functions of their protein products. The fundamental mechanisms of transcriptional regulation in bacteria were worked out in this way ${ }^{12}$.

as transcriptional regulators. Mutations in yeast Srb genes, unlike TAF mutations, generate global defects in class II gene expression and affect their ability to respond to regulators in vivo. Purified yeast RNA polymerase II holoenzyme can respond to activators in vitro in the absence of TAFs.

In this context, it is possible that the TAFs are indeed generally involved in the response to activators in vivo but are ancillary to the holoenzyme at most promoters. Activators can interact in vitro with components of the holoenzyme as well as $\mathrm{TAFs}^{5,6}$. Contact between artificial activators and almost any component of the transcription-initiation apparatus is sufficient to activate transcription in vivo $o^{7,8}$.

Another possibility is that the TAFs function at only a small number of promoters in vivo. Struhl and colleagues ${ }^{4}$ found two genes whose rate of transcription decreases when expression of some TAFs is shut down in vivo. It is intriguing that the promoters of both these genes have TATA boxes whose sequence deviates from the consensus. TAFs are required for transcription from such promoters in vitro; furthermore, at least one TAF binds directly to core promoter elements expected to be important for guiding transcription from promoters lacking consensus TATA boxes ${ }^{1,2}$.
But each approach has its limitations. Reconstituted biochemical reactions are usually a highly simplified version of what goes on inside a living cell. The reaction may be missing essential features of the process as it occurs in vivo (which may be poorly defined) or it may harbour hidden flaws - for example, TFIIC is missing from the roll call of general transcription factors because its identification was an artefact of the in vitro assay conditions.

The problem for geneticists is that the genes identified in genetic selections may not always be directly involved in the process of interest. Thus, the general transcription factor TFIIB was inadvertently identified in yeast by a screen intended to isolate translation factors. Other transcription factors have been isolated in screens for cell-cycle regulators because of their indirect effects on the expression of these regulators.

Some of these drawbacks can be overcome by sharing tactics, as we have seen in the transcription story. The chief weakness of biochemistry - inability to discriminate between what is real and what is artefact - can be addressed genetically. A biochemist with a newly purified enzyme can mutate the gene encoding that enzyme. If the biochemist is correct about the enzyme's activity, then mutation of the gene should affect the cell's

The observation that inactivation or elimination of TAFs is lethal to cells suggests that TAFs are critical for the expression of certain essential genes. TAFs appear to be involved in cell-cycle progression in yeast and mammalian cells. Green and colleagues ${ }^{3}$ point out that inactivation of particular yeast TAFs can cause growth arrest at distinct stages in the cell cycle. One of their temperature-sensitive yeast TAF $_{\text {II }} 145$ mutants has a phenotype remarkably similar to that found in mammalian cells with a mutation in the homologue $\mathrm{TAF}_{\mathrm{II}} 250$; the mammalian temperature-sensitive cell line arrests growth at the G1 stage of the cell cycle when the temperature is raised, but does not exhibit a global defect in class II gene transcription (ref. 9 and references therein).

Transcription in yeast and mammalian cells almost certainly follows the same fundamental principles, but there is no doubt that mammalian cells need to respond to more complicated and diverse regulatory signals. For example, whereas yeast have only a few developmental programmes, mammals have several hundred. If TAFs are necessary for integrating responses to diverse regulatory controls such as those governing the cell cycle and cell fate, they may be essential for expression of a higher proportion of genes in ability to perform the reaction concerned. Likewise, the chief weakness of genetics - inability to distinguish direct from indirect effects - can be tackled biochemically. A geneticist with a newly identified gene can set up assays to determine the enzymatic properties of the protein encoded by the gene. If the gene does what the geneticist thinks it does in vivo, then the protein encoded by that gene should have the proper enzymatic activity in vitro.

Of course, combining biochemistry and genetics is not always so straightforward. In some experimental animal systems, genetic manipulation techniques are not yet advanced enough to allow specific mutations to be made in a particular gene. Also, there are often difficulties in developing biochemical assays for biological processes that have not been fully defined in vivo.

These challenges have been met, at least in part, by molecular biologists, who use recombinant DNA technology to isolate, mutate and analyse genes and their protein products: molecular-biological tools help to bridge the gap between biochemistry and genetics. Indeed, it is now the combination of biochemistry, genetics and molecular biology that drives progress in biology, each providing checks and balances on the others. $\quad$ D.M.C. \& R.A.Y.

mammalian cells than in yeast.

The final textbook entry explaining exactly how and where TAFs function in vivo remains to be written. Whatever the outcome, however, the history of gene-activation research underscores the importance of combining genetic and biochemical approaches.

David M. Chao and Richard A. Young are in the Whitehead Institute for Biomedical Research, Nine Cambridge Center, Cambridge, Massachusetts 02142, and the Department of Biology, Massachusetts Institute of Technology, Cambridge, Massachusetts 02139, USA.

\footnotetext{
1. Goodrich, J. A., Cutler, G. \& Tjian, R. Cel/ 84, 825-830 (1996)

Burley, S. K. \& Roeder, R. G. Annu. Rev. Biochem. 65 769-799 (1996)

Walker, S. S., Reese, J. C., Apone, L. M. \& Green, M. R. Nature 383, 185-188 (1996)

Moqtaderi, Z , Bai, Y. Poon, D., Weil, P. A. \& Struhl, K. Nature 383, 188-191 (1996)

Koleske, A. \& Young R. A. Trends Biochem. Sci. 20 113-116 (1995).

6. Halle, J.P. \& Meisterernst, M. Trends Genet. 12 161-163 (1995)

Barberis, A. et al. Cell 81, 359-368 (1995)

8. Struhl, K. Cell 84, 179-182 (1996)

9. Wang, E. H. \& Tjian, R. Science 263, 811-814 (1994).

10. Conaway, R. C. \& Conaway, J. W. Annu. Rev. Biochem. 62, 161-190 (1993).

11. Zawel, L. \& Reinberg, D. Annu. Rev. Biochem. 64, 533-561 (1995)

12. Jacob, F. \& Monod, J. J. Mol. Biol. 3, 318-356 (1961).
} 